\title{
Trends in Coronavirus Disease 2019 Hospitalization and Prognosis: Gender Effect*
}

\author{
Mei-jing SHI ${ }^{1,2}$, Jia-gao LV${ }^{1}$, Li LIN ${ }^{1}$, Jun-yi GUO ${ }^{1 \#}$ \\ ${ }^{1}$ Division of Cardiology, Department of Internal Medicine, Tongji Hospital, Tongji Medical College, Huazhong University of \\ Science and Technology, Wuhan 430030, China \\ ${ }^{2}$ Department of Emergence, General Hospital of the Yangtze River Shipping, Wuhan 430030, China
}

(C) Huazhong University of Science and Technology 2021

\begin{abstract}
Summary: We here aimed to investigate the impact of gender on the clinical characteristics and laboratory results of patients with coronavirus disease 2019 (COVID-19) and provide clues to the pathological mechanisms underlying COVID-19. A retrospective study was performed. Clinical characteristics, severity of lung infection, laboratory results, and prognoses of patients of different gender were analyzed. A total of 242 patients were finally included. The median age was 58 years (IQR: 40-68), including 54 (22.3\%) hospital staffs. Ninety-four (38.8\%) were male and $148(61.1 \%)$ were female. The proportion of patients with diabetes was significantly higher in the male group than in the female group $(P=0.034)$. Male patients had a significantly larger proportion of severe lung infection, higher leukocyte count, neutrophil count, neutrophil-to-lymphocyte ratio, C-reactive protein, and procalcitonin than female. Furthermore, male patients had worse liver, cardiac, and coagulation function than their female counterparts. Male patients with COVID-19 showed more severe inflammation reaction and coagulation dysfunction than female patients. In conclusion, gender is associated with host response to SARS-CoV-2 infection.
\end{abstract}

Key words: COVID-19; gender; blood routine; coagulation function; inflammation

Coronavirus disease 2019 (COVID-19) has become a major public health threat around the world. From its outbreak through April 3, 2021, SARS-CoV2 caused over 129 million confirmed infections with over 2831815 deaths worldwide. The clinical characteristics of COVID-19 patients have been widely reported ${ }^{[1-3]}$. Although current data for COVID-19 show equal numbers of cases between men and women, men seem to be more at risk for worse outcomes and death than women. Jin et al analyzed the public dataset of the first 37 patients who died of COVID-19 in China and 1019 patients who survived. They reported that the number of men who died from COVID-19 to

Mei-jing SHI, E-mail: smjmedcine@163.com

\#Corresponding author, E-mail: gjytjmu@163.com

${ }^{*}$ This work was supported by the National Natural Science Foundation of China (Nos. 81570416, 81570337), Hubei Province Health and Family Planning Scientific Research Project (No. WJ2019M120), Natural Science Foundation of Hubei Province (No. 2019CFB668), the Fundamental Research Fund for the Central Universities (HUST) (No. 2017KFYXJJ099), the Science and Technology Project Foundation of Wuhan (No. 2017060201010175), and the Outstanding Young Investigator Foundation of Tongji Hospital (No. YXQN009). be 2.4 times that of women ${ }^{[4]}$. Similarly, a study of 72314 patients with COVID-19 in China found that while men and women had the same prevalence, the mortality rate of male patients (653/22 981) was higher than female patients $(370 / 21691)^{[5]}$. Besides, a recent published meta-analysis which included a total of 33934 males and 32969 females exhibited more severe symptoms and a worse prognosis in males than in females ${ }^{[6]}$. However, the current studies only provided data grouped by the severity of diseases, so the specific impact of gender on the progression of COVID-19 remains uncertain. Determining the differences in the clinical manifestations and laboratory tests between patients of different gender might provide clues to the pathogenesis of COVID-19[7].

This study aims to provide information about the specific impact of gender on the clinical manifestations, laboratory tests, and prognoses of patients with COVID-19 in Wuhan.

\section{MATERIALS AND METHODS}

\subsection{Data Source and Study Design}

We performed a retrospective real-world database analysis which complied with the Declaration of 
Helsinki and was approved by the Ethical Review Board of General Hospital of Yangtze River Shipping (LW2020026). Written informed consent was not obtained because the data were analyzed retrospectively and anonymously. In total, 285 consecutive COVID-19 confirmed patients admitted to General Hospital of Yangtze River Shipping between January 9, 2020 and March 6, 2020 were included in this study. Exclusion criteria included: (1) referral to other hospitals $(n=35)$, (2) incomplete medical records $(n=7)$, or (3) in a persistent vegetative state before infection $(n=1)$. Our final study included 242 patients, among whom 227 were survivors and 15 were dead. The gender, age, past-history, vital signs, severity of CT scan, blood routine test, liver function, coagulation function, C-reactive protein (CRP), procalcitonin (PCT), blood lipids, and myocardial injury markers were retrieved from the medical records and electronic databases of General Hospital of Yangtze River Shipping, Wuhan, China on March 7th, 2020.

\subsection{The Diagnosis and Severity of COVID-19}

COVID-19 was confirmed by the history of epidemiology, clinical manifestations, CT imaging, and the nucleic acid detection (NAT) according to the Diagnosis and Treatment Program of 2019 New Coronavirus Pneumonia (trial seventh version) by National Health and Health Commission of China in $2020^{[8]}$. The severity of COVID-19 was assessed based on the most serious conditions the patients ever experienced according to the WHO classification of COVID-19 severity ${ }^{[9]}$.

\subsection{Severity of Lung Injury by CT Scan}

Chest CT scans were performed in the outpatient, at admission, once weekly after admission until they were discharged from the hospital. Each patient had at least four chest $\mathrm{CT}$ scan tests. The severity of lung injury was evaluated based on the chest CT scan. Based on the staging criteria of the Guideline for Imaging Diagnosis of Novel Coronavirus (2019-nCoV) Infected Pneumonia (2nd edition 2020) ${ }^{[10]}$, we defined mild case as: single or multiple lung subpleural plaques or ground-glass opacity (GGO) under the center of the lobes; moderate lung injury case as: large range of new and old GGO, the reality changes, but it does not reach the standard of heavy weight. Severe lung injury case was defined as multiple GGO in both lungs, diffuse multiple large consolidations, combined with thickening of the lobular septum and pleural septum of the lung, with or without pleural effusion on one side.

\subsection{Statistical Analyses}

The data were analyzed by SPSS version 24.0 for Mac (SPSS Inc., USA). Continuous variables with normal distribution were presented as mean $\pm \mathrm{SD}$. Nonnormal variables were reported as median (Q1-Q3 quartiles). The normality of distribution of continuous variables was tested by the one-sample Kolmogorov-
Smirnov test. Categorical variables were expressed as number (percentage). Means of 2 continuous normally distributed variables were compared by independent samples Student's $t$-test. Mann-Whitney $U$ test was run to determine if there were differences between two non-normal variables. The frequencies of categorical variables were compared using Pearson $\chi^{2}$ (with or without continuity correction), Fisher's exact test, or Mantel-Haenszel Test, when appropriate. Some covariates had missing values, and we applied max likelihood by expectation maximization and built imputation data to replace the missing values. The multivariate binary logistic regression analysis was used to ascertain the association between gender and patients' prognosis after adjusting for the WHO verified risk factors (age more than 60 years, diabetes, hypertension, and cardiac disease). A $P$-value of $<0.05$ was set as the level of statistical significance.

\section{RESULTS}

\subsection{Comparison of Basic Clinical Characteristics between Patients with Different Gender}

As shown in table 1, a total of 242 patients were finally included in this study. The median age was 58 years (IQR: 40-68), including 54 (22.3\%) hospital staffs. Ninety-four (38.8\%) were male and $148(61.1 \%)$ were female. Here, $27(11.2 \%)$ patients had diabetes, $75(31.0 \%)$ patients had a history of hypertension, 21 $(8.7 \%)$ had coronary artery disease (CAD), 3 (1.2\%) had heart failure, and $12(5.0 \%)$ had arrhythmia. Male patients were more likely to have diabetes than their female counterparts $(P=0.034)$ and also had higher heart rate $(P=0.009)$. There were no differences in other factors between the two groups.

\subsection{Comparison of Chest CT and Laboratory Results of Patients Grouped by Gender}

As shown in table 2, 128 (52.9\%), 56 (23.1\%), and $58(24.0 \%)$ patients had mild, moderate, and severe lung infection, respectively. Lung infection was more severe in male than in female patients $(P=0.002)$. Male patients also had significantly higher level of hemoglobin concentration $(P<0.001)$, leukocyte count $(P<0.001)$, neutrophil count $(P<0.001)$, and neutrophil-to-lymphocyte ratio (NLR) $(P<0.001)$ than female patients. There were significantly higher levels of ALT $(P<0.001)$, direct bilirubin $(P<0.001)$, and indirect bilirubin $(P<0.001)$ in male patients. Concerning coagulation function parameters, prothrombin time (PT) was significantly higher in male patients $(P<0.001)$. As for infection-related parameters, both $\mathrm{C}$-reactive protein $(\mathrm{CRP})(P<0.001)$ and procalcitonin (PCT) $(P=0.002)$ were significantly higher in the male group than in the female group. Male patients had significantly lower levels of high-density lipoprotein (HDL) than female patients $(P=0.001)$. 
Table 1 Comparison of basic conditions of patients of different gender

\begin{tabular}{|c|c|c|c|c|}
\hline Parameter & All patients $(n=242)$ & Male $(n=94)$ & Female $(n=148)$ & $P$ value \\
\hline Age (years) & $58(40,68)$ & $56 \pm 18$ & $55(38,68)$ & 0.567 \\
\hline$<40$ & $64(26.4 \%)$ & $23(24.5 \%)$ & $23(24.5 \%)$ & 0.843 \\
\hline $40-60$ & $72(29.8 \%)$ & $29(30.9 \%)$ & $29(30.9 \%)$ & \\
\hline $60-70$ & $60(24.8 \%)$ & $22(23.4 \%)$ & $22(23.4 \%)$ & \\
\hline$\geq 70$ & $46(19.0 \%)$ & $20(21.3 \%)$ & $20(21.3 \%)$ & \\
\hline Diabetes & $27(11.2 \%)$ & $16(17.0 \%)$ & $11(7.4 \%)$ & $0.034^{*}$ \\
\hline \multicolumn{5}{|c|}{ Cardiovascular complications } \\
\hline Hypertension & $75(31.0 \%)$ & $33(35.1 \%)$ & $42(28.4 \%)$ & 0.270 \\
\hline CAD & $21(8.7 \%)$ & $11(11.7 \%)$ & $10(6.8 \%)$ & 0.183 \\
\hline Heart failure & $3(1.2 \%)$ & $2(2.1 \%)$ & $1(0.7 \%)$ & 0.562 \\
\hline Arrhythmia & $12(5.0 \%)$ & $7(7.4 \%)$ & $5(3.4 \%)$ & 0.155 \\
\hline Hospital staff & $54(22.3 \%)$ & $18(19.1 \%)$ & $36(24.3 \%)$ & 0.346 \\
\hline \multicolumn{5}{|l|}{ Vital signs } \\
\hline Body temperature $\left({ }^{\circ} \mathrm{C}\right)$ & $38.2(37.0,38.7)$ & $38.4(37.0,38.8)$ & $38.0(37.0,38.6)$ & 0.058 \\
\hline $\mathrm{SBP}(\mathrm{mmHg})$ & $122(120,130)$ & $122(120,130)$ & $120(120,130)$ & 0.802 \\
\hline $\mathrm{DBP}(\mathrm{mmHg})$ & $76(70,80)$ & $76(70,80)$ & $76(70,80)$ & 0.964 \\
\hline HR (bpm) & $82(78,90)$ & $85(79,93)$ & $80(78,88)$ & $0.009^{*}$ \\
\hline $\mathrm{SaO}_{2}(\%)$ & $98(96,99)$ & $98(94,99)$ & $98(97,99)$ & 0.065 \\
\hline
\end{tabular}

$\mathrm{CAD}$, cardiovascular artery disease; $\mathrm{SBP}$, systolic blood pressure; $\mathrm{DBP}$, diastolic blood pressure; HR, heart rate; $\mathrm{SaO}_{2}$, oxygen saturation; ${ }^{*} P<0.05$

Notably, significantly more patients in the male group had abnormal levels of NT-proBNP $(P<0.001)$. Male patients had significantly higher levels of cTnl than female patients $(P<0.001)$.

\subsection{Prognoses of Patients of Different Gender and Age}

As shown in table 3, more patients in the male group $(11.7 \%$ vs. $3.4 \%, P=0.011)$ received mechanical ventilation than those in the female group. Male patients also had significantly higher rates of the severe type of COVID-19 than females did $(P=0.003)$. To ascertain the association between gender and patients' prognosis, a multivariate binary logistic regression analysis was performed. After adjusting for the available WHO verified risk factors (age more than 60 years, diabetes, hypertension, and cardiac disease), gender was still significantly associated with the use of mechanical ventilation $(P=0.037)$, severe type of COVID-19 $(P=0.026)$ and in-hospital death $(P=0.019)$.

\section{DISCUSSION}

In this retrospective cohort study, we assessed the impact of gender on the clinical characteristics and prognoses of patients with COVID-19 in Wuhan. A total of 242 patients were finally included in this study. The proportion of patients with diabetes was significantly higher in the male group than in the female group $(P=0.034)$. Male patients had significantly higher rates of severe lung infection, higher levels of hemoglobin concentration, leukocyte count, neutrophil count, and NLR than their female counterparts. The male patients had worse liver, cardiac, and coagulation function than the female patients. Both CRP and PCT levels were significantly higher in the male group than in the female group.

Although current data for COVID-19 show equal number of cases between men and women, men seem to be more at risk for worse outcomes and death than women $^{[11]}$. However, it is not clear yet why males have suffered higher mortality from COVID-19. Men and women differ substantially regarding genetics, hormonal milieu, immune function, neurocognitive aging process, vascular health, response to therapeutics, and interaction with health care systems. The impact of these above factors on the association between gender and prognosis of patients was widely discussed in recent reviews ${ }^{[12-14]}$. Collecting gender-disaggregated data is important to improve care for COVID-19 patients $^{[7]}$. Our study provides information about the specific impact of gender on the clinical manifestations, laboratory tests, and prognoses of patients with COVID-19 in Wuhan, which might provide clues to the pathogenesis of COVID-19.

At present, it is widely believed that pre-existing conditions, including diabetes, CAD, hypertension, and obesity, are associated with poorer outcomes of COVID-19[15, 16]. We found that the proportion of patients with diabetes was significantly higher in the male group than in the female group and the cardiovascular complications were also more common in males than in females, which might support the hypothesis that the better outcomes of COVID-19 were due to the lower burden of comorbidity in female patients $^{[17]}$.

Studies have shown that gender, here understood in its physical sense, contributes significantly to immune response ${ }^{[18]}$, which is considered to play 
Table 2 Comparison of 128-slice CT and laboratory indicators in patients of different gender

\begin{tabular}{|c|c|c|c|c|}
\hline Parameter & All patients $(n=242)$ & Male $(n=94)$ & Female $(n=148)$ & $P$ value \\
\hline Severity of lung infection by CT scan & & & & $0.002^{*}$ \\
\hline Mild & $128(52.9 \%)$ & $63(67.0 \%)$ & $124(83.8 \%)$ & \\
\hline Moderate & $56(23.1 \%)$ & $18(19.1 \%)$ & $19(12.8 \%)$ & \\
\hline Severe & $58(24.0 \%)$ & $13(13.8 \%)$ & $5(3.4 \%)$ & \\
\hline \multicolumn{5}{|l|}{ Blood routine test } \\
\hline $\mathrm{Hb}(\mathrm{g} / \mathrm{L})$ & $118(108,131)$ & $126(118,139)$ & $113(104,124)$ & $<0.001^{*}$ \\
\hline Plt $\left(\times 10^{9} / \mathrm{L}\right)$ & $161 \pm 58$ & $157 \pm 59$ & $161(126,200)$ & 0.821 \\
\hline $\mathrm{WBC}\left(\times 10^{9} / \mathrm{L}\right)$ & $5.06(3.88,6.90)$ & $5.8(4.6,7.9)$ & $4.6(3.6,6.0)$ & $<0.001^{*}$ \\
\hline Neutrophil $\left(\times 10^{9} / \mathrm{L}\right)$ & $3.10(2.22,4.96)$ & $3.9(2.8,6.6)$ & $2.8(2.0,3.9)$ & $<0.001^{*}$ \\
\hline Lymphocyte $\left(\times 10^{9} / \mathrm{L}\right)$ & $1.15(0.80,1.53)$ & $1.1(0.7,1.5)$ & $1.2(0.9,1.5)$ & 0.107 \\
\hline NLR & $2.6(1.6,5.1)$ & $3.2(2.0,9.1)$ & $2.1(1.6,3.7)$ & $<0.001^{*}$ \\
\hline \multicolumn{5}{|l|}{ Liver function } \\
\hline ALT (U/L) & $30(17,51)$ & $39(23,74)$ & $26(15,44)$ & $<0.001^{*}$ \\
\hline Total bilirubin $(\mu \mathrm{mol} / \mathrm{L})$ & $11.27(8.04,16.29)$ & $13.52(9.49,18.77)$ & $10.14(7.48,14.98)$ & $<0.001^{*}$ \\
\hline Direct bilirubin $(\mu \mathrm{mol} / \mathrm{L})$ & $2.17(1.49,3.57)$ & $3.07(2.07,4.51)$ & $1.68(1.25,2.53)$ & $<0.001^{*}$ \\
\hline Indirect bilirubin $(\mu \mathrm{mol} / \mathrm{L})$ & $8.85(6.36,12.87)$ & $10.14(7.07,14.98)$ & $8.27(6.03,11.48)$ & $0.011^{*}$ \\
\hline \multicolumn{5}{|l|}{ Coagulation function } \\
\hline PT (s) & $11.4(10.9,11.9)$ & $12(11,12)$ & $11(11,12)$ & $<0.001^{*}$ \\
\hline D-dimer ( $\mu \mathrm{g} / \mathrm{mL}$ FEU) & $0.49(0.24,1.47)$ & $0.49(0.24,1.54)$ & $0.49(0.23,1.34)$ & 0.606 \\
\hline \multicolumn{5}{|l|}{ Inflammatory indicators } \\
\hline $\mathrm{CRP}(\mathrm{mg} / \mathrm{L})$ & $11.5(2.10,30.3)$ & $24.90(3.20,33.05)$ & $6.95(1.70,23.38)$ & $<0.001^{*}$ \\
\hline $\operatorname{PCT}(\mu \mathrm{g} / \mathrm{L})$ & $0.10(0.10,0.10)$ & $0.10(0.10,0.10)$ & $0.10(0.10,0.10)$ & $0.002^{*}$ \\
\hline \multicolumn{5}{|l|}{ Blood lipids } \\
\hline $\mathrm{TC}(\mathrm{mmol} / \mathrm{L})$ & $4.24(3.65-5.01)$ & $4.26 \pm 1.00$ & $4.25(3.81-5.12)$ & 0.224 \\
\hline $\mathrm{TG}(\mathrm{mmol} / \mathrm{L})$ & $1.19(0.82-1.71)$ & $1.20(0.86-1.72)$ & $1.19(0.81-1.70)$ & 0.628 \\
\hline $\mathrm{HDL}(\mathrm{mmol} / \mathrm{L})$ & $1.20(0.96-1.39)$ & $1.11 \pm 0.27$ & $1.26 \pm 0.36$ & 0.001 \\
\hline $\mathrm{LDL}(\mathrm{mmol} / \mathrm{L})$ & $2.68(2.19-3.44)$ & $2.68(215-3.53)$ & $2.68(2.21-3.33)$ & 0.998 \\
\hline \multicolumn{5}{|l|}{ Myocardial injury markers } \\
\hline $\operatorname{AST}(\mathrm{U} / \mathrm{L})$ & $27(19,41)$ & $33(21,52)$ & $24(17,38)$ & $0.001^{*}$ \\
\hline LDH (U/L) & $205(177,274)$ & $222(193,304)$ & $202(168,234)$ & $0.001^{*}$ \\
\hline NT-proBNP $(p g / m L)$ & $102(72,169)$ & $102.0(66.2,227.0)$ & $102.0(79.3,140.4)$ & 0.776 \\
\hline NT-proBNP $>125 \mathrm{pg} / \mathrm{mL}$ & $66(27.3 \%)$ & $26(27.7 \%)$ & $19(12.8 \%)$ & $0.004^{*}$ \\
\hline cTnl (ng/L) & $7.03(4.76,11.19)$ & $8(6,17)$ & $7(4,9)$ & $<0.001^{*}$ \\
\hline $\mathrm{cTnl}>14 \mathrm{ng} / \mathrm{L}$ & $45(18.6 \%)$ & $29(30.9 \%)$ & $37(25.0 \%)$ & 0.319 \\
\hline
\end{tabular}

$\mathrm{Hb}$, hemoglobin; Plt, platelet; WBC, white blood cell; NLR, neutrophil-to-lymphocyte ratio; ALT, aminoleucine transferase; PT: prothrombin time; CRP: C-reactive protein; PCT: procalcitonin; TC, total cholesterol; TG, total triglyeride; HDL, high-density lipoprotein; LDL, low-density lipoprotein; AST, aspartate transaminase; LDH, lactate dehydrogenase; NT-proBNP, N-terminal prohormone of brain natriuretic peptide; cTnI, cardiac troponin I ; FEU, fibrinogen equivalent units; ${ }^{*} P<0.05$

Table 3 Comparison of severity of COVID-19 and prognosis in patients of different gender

\begin{tabular}{lccccc}
\hline Parameter & All patients $(n=242)$ & Male $(n=94)$ & Female $(n=148)$ & $P$ value & Adjusted $P$ value \\
\hline Mechanical ventilation & $17(7.0 \%)$ & $11(11.7 \%)$ & $5(3.4 \%)$ & 0.011 & $0.037^{*}$ \\
COVID-19 severity & & & & 0.003 & $0.026^{*}$ \\
$\quad$ Moderate disease & $202(83.5 \%)$ & $71(75.5 \%)$ & $131(88.5 \%)$ & & \\
$\quad$ Severe disease & $23(9.5 \%)$ & $10(10.6 \%)$ & $13(8.8 \%)$ & & \\
$\quad$ Critical disease & $17(7.0 \%)$ & $13(13.8 \%)$ & $4(2.7 \%)$ & & $0.019^{*}$ \\
Prognosis & & & & \\
$\quad$ Healed and discharged & $227(93.8 \%)$ & $83(88.3 \%)$ & $144(97.3 \%)$ & & \\
$\quad$ Death & $15(6.2 \%)$ & $11(11.7 \%)$ & $4(2.7 \%)$ & & \\
\hline
\end{tabular}

an important role in the prognosis of COVID-19 ${ }^{[19]}$. Although, for most patients, the laboratory findings were still within normal ranges in our study, we found that male patients had higher leukocyte count and neutrophil count than female patients. In fact, the blood cell count also showed sexual difference in healthy adults ${ }^{[20]}$ and the white blood cell count was higher in males than in females. However, the difference was not significant enough to have clinical significance ${ }^{[21]}$. The infection of SARS-CoV-2 might exaggerate the variation in leukocyte count between males and females. It has been reported that elevated 
NLR, CRP and PCT are associated with poor clinic outcomes of COVID-1 $9^{[22,23]}$. We found that NLR, CRP and PCT were significantly higher in male patients, which might indicate that gender also influenced the immune response to the infection of SARS-CoV-2. We found that male patients developed liver function impairment more easily, with significantly increased levels of ALT, direct bilirubin, and indirect bilirubin. They were also more likely to suffer from myocardial injury. Liver and cardiac dysfunction might be secondary to hyperinflammation and cytokine storms in COVID-19 ${ }^{[24,25]}$, which might be greater in magnitude in male patients.

Several studies have identified a link between severe COVID-19 patients and specific coagulation index, in particular, high D-dimer level, and prolonged $\mathrm{PT}^{[26]}$. While healthy females and males show similar $\mathrm{PT}^{[27]}$, we found that $\mathrm{PT}$ was significantly higher in male patients. The increased PT might also contribute to the worse outcome in male patients.

This study has several limitations. First, this was a single-center study, so it may lack representativeness. The sample size was also relatively small. A multicenter cohort study should be carried out to validate this outcome. Second, some scholars believe that the higher mortality in male patients might be associated with the much higher rate of smoking among men than in women in China (288 million men vs. 12.6 million women were reported to be smokers in 2018) ${ }^{[28]}$. However, we have no data on patients' smoking history. At present, we still do not understand the molecular basis of severe COVID-19 symptoms, so research is urgently needed to identify biomarkers that could enable early identification of high-risk individuals.

In summary, our study indicates that gender was associated with the host response to SARSCoV-2 infection. The specific difference in clinical characteristics between male patients and their female counterparts included higher co-existence of basic diseases, abnormal blood routine test results, coagulation function, and inflammatory parameters. Our study might provide clues to the pathologic mechanisms underlying COVID-19.

\section{Conflict of Interest Statement}

The authors declare that there is no conflict of interest with any financial organization or corporation or individual that can inappropriately influence this work.

\section{REFERENCES}

1 Guan WJ, Ni ZY, Hu Y, et al. Clinical characteristics of coronavirus disease 2019 in China. N Engl J Med, 2020,382(18):1708-1720

2 Zhou F, Yu T, Du R, et al. Clinical course and risk factors for mortality of adult inpatients with COVID-19 in wuhan, China: A retrospective cohort study. Lancet, 2020,395(10229):1054-1062
3 Huang C, Wang Y, Li X, et al. Clinical features of patients infected with 2019 novel coronavirus in wuhan, China. Lancet, 2020, 395(10223):497-506

4 Jin JM, Bai P, He W, et al. Gender differences in patients with COVID-19: Focus on severity and mortality. Front Public Health 2020,8(152):1-6

5 Epidemiology working Group for NCIP Epidemic Response, Chinese Center for Disease Control and Prevention. The epidemiological characteristics of an outbreak of 2019 novel coronavirus diseases (COVID-19) in China. Zhonghua Liu Xing Bing Xue Za Zhi (Chinese), 2020,41(2):145-151

6 Ortolan A, Lorenzin M, Felicetti M, et al. Does gender influence clinical expression and disease outcomes in COVID-19? A systematic review and meta-analysis. Int J Infect Dis, 2020,99:496-504

7 Spagnolo PA, Manson JE, Joffe H. Sex and gender differences in health: What the COVID-19 pandemic can teach us. Ann Intern Med, 2020,173(5):385-386

8 Diagnosis and treatment program of 2019 new coronavirus pneumonia (trial seventh version). (Chinese). 2020 (http://www.nhc.gov.cn/xcs/zhengcwj/ 202003/46c9294a7dfe4cef80dc7f5912eb1989/files/ce3 e6945832a438eaae415350a8ce964.pdf)

9 Clinical management of COVID-19. 2020 (https://apps. who.int/iris/rest/bitstreams/1278777/retrieve)

10 Chinese Research Hospital Association Standing Committee on Infection and Inflammation, et al. Guideline for imaging diagnosis of novel coronavirus(2019-ncov) infected pneumonia (2nd edition 2020). Shoudu Yike Daxue Xuebao (Chinese). 2020,41(2):168-173

11 Mohamed MO, Gale CP, Kontopantelis E, et al. Sex differences in mortality rates and underlying conditions for COVID-19 deaths in england and wales. Mayo Clin Proc, 2020,95(10):2110-2124

12 Anca PS, Toth PP, Kempler P, et al. Gender differences in the battle against COVID-19: Impact of genetics, comorbidities, inflammation and lifestyle on differences in outcomes. Int J Clin Pract, 2020,75(2):e13666

13 Haitao T, Vermunt JV, Abeykoon J, et al. COVID-19 and sex differences: Mechanisms and biomarkers. Mayo Clin Proc, 2020,95(10):2189-2203

14 Li Y, Jerkic M, Slutsky AS, et al. Molecular mechanisms of sex bias differences in COVID-19 mortality. Crit Car, 2020,24(1):405

15 Guan WJ, Liang WH, Zhao Y, et al. Comorbidity and its impact on 1590 patients with COVID-19 in China: A nationwide analysis. Eur Respir J, 2020,55(5):2000547

16 Lauc G, Sinclair D. Biomarkers of biological age as predictors of COVID-19 disease severity. Aging (Albany NY), 2020,12(8):6490-6491

17 The Lancet. The gendered dimensions of COVID-19. Lancet, 2020,395(10231):1168

18 Markle JG, Fish EN. Sex matters in immunity. Trends Immunol, 2014,35(3):97-104

19 Maggi E, Canonica GW, Moretta L. COVID-19: Unanswered questions on immune response and pathogenesis. J Allergy Clin Immunol, 2020,146(1):1822

20 Wakeman L, Al-Ismail S, Benton A, et al. Robust, routine haematology reference ranges for healthy adults. 
Int J Lab Hematol, 2007,29(4):279-283

21 Qiao R, Yang S, Yao B, et al. Complete blood count reference intervals and age- and sex-related trends of north China han population. Clin Chem Lab Med, 2014,52(7):1025-1032

22 Qin C, Zhou L, Hu Z, et al. Dysregulation of immune response in patients with COVID-19 in wuhan, China. Clin Infect Dis. 2020,71(15):762-768

23 Terpos E, Ntanasis-Stathopoulos I, Elalamy I, et al. Hematological findings and complications of COVID-19. Am J Hematol. 2020,95(7):834-847

24 Akhmerov A, Marban E. COVID-19 and the heart. Circ Res. 2020,126(10):1443-1455

25 Tay MZ, Poh CM, Renia L, et al. The trinity of
COVID-19: Immunity, inflammation and intervention. Nat Rev Immunol. 2020,20(6):363-374

26 Salamanna F, Maglio M, Landini MP, et al. Platelet functions and activities as potential hematologic parameters related to coronavirus disease 2019 (COVID-19). Platelets, 2020,31(5):627-632.

27 Weidhofer C, Meyer E, Ristl R, et al. Dynamic reference intervals for coagulation parameters from infancy to adolescence. Clin Chim Acta. 2018,482:124-135

28 Cai H. Sex difference and smoking predisposition in patients with COVID-19. Lancet Respir Med, 2020,8(4): e20

(Received Oct. 28, 2020; accepted Jan. 27, 2021) 